\title{
Geodesics on tangent bundles with horizontal Sasaki gradient metric
}

\author{
Abderrahim Zagane
}

Received: 23.06.2020 / Revised: 04.08.2020 / Accepted: 18.10.2020

\begin{abstract}
Let $\left(M^{m}, g\right)$ be an $m$-dimensional Riemannian manifold and $T M$ be its tangent bundle. In this paper, we introduce a new class of natural metrics with respect to g non-rigid on $T M$, called the horizontal Sasaki gradient metric. we investigate geodesics of the horizontal Sasaki gradient metric. Afterward establish a necessary and sufficient conditions under which a curve be a geodesic respect.
\end{abstract}

Keywords. Horizontal lift, vertical lift, horizontal Sasaki gradient metric, geodesic.

Mathematics Subject Classification (2010): 53C22, 58E10, 53C20.

\section{Introduction}

The geometry of the tangent bundle $T M$ equipped with Sasaki metric has been studied by many authors Sasaki S. [13], Yano K. and Ishihara S. [15], Dombrowski P. [5] , Cengiz N. and Salimov A.A [3], Salimov A.A and Gezer A. [10]. Also, other further signifiant developments are due in some surveys, [9], [11] etc... The rigidity of Sasaki metric has incited some geometers to construct and study other metrics on $T M$. Musso E. and Tricerri F. [8] has introced the notion of Cheeger-Gromoll metric, this metric has been studied also by many authors (see [1], [4], [6], [12],[14]). Other new works have appeared in the articles [2],[7], [16], [17].

The main idea in this note consists in the modification of the Sasaki metric. First we introduce a new metric called horizontal Sasaki gradient metric on the tangent bundle $T M$, then we establish the Levi-Civita connection of this metric (Theorem 3.1) and we establish a necessary and sufficient conditions under which a curve be a geodesic with respect to the horizontal Sasaki gradient metric (Theorem 4.2, Corollary 4.1, Corollary 4.2 and Corollary 4.3). Afterward we investigate some properties of geodesic (Theorem 4.3, Theorem 4.4 and Corollary 4.5). Next we also construct some examples of geodesics ( Example 1 and Example 2).

\section{Preliminaries}

Let $\left(M^{m}, g\right)$ be an m-dimensional Riemannian manifold and $(T M, \pi, M)$ be its tangent bundle. A local chart $\left(U, x^{i}\right)_{i=\overline{1, m}}$ on $M$ induces a local chart $\left(\pi^{-1}(U), x^{i}, y^{i}\right)_{i=1, m}$ on $T M$. Denote by $\Gamma_{i j}^{k}$ the Christoffel symbols of $g$ and by $\nabla$ the Levi-Civita connection of $g$.

A. Zagane

University Center Ahmed Zabana-Relizane, Dept. of mathematics, Relizane-Algeria

E-mail: Zaganeabr2018@gmail.com 
We have two complementary distributions on $T M$, the vertical distribution $\mathcal{V}$ and the horizontal distribution $\mathcal{H}$, defined by :

$$
\begin{gathered}
\mathcal{V}_{(x, u)}=\operatorname{Ker}\left(d \pi_{(x, u)}\right)=\left\{\left.a^{i} \frac{\partial}{\partial y^{i}}\right|_{(x, u)}, a^{i} \in \mathbb{R}\right\}, \\
\mathcal{H}_{(x, u)}=\left\{\left.a^{i} \frac{\partial}{\partial x^{i}}\right|_{(x, u)}-\left.a^{i} u^{j} \Gamma_{i j}^{k} \frac{\partial}{\partial y^{k}}\right|_{(x, u)}, a^{i} \in \mathbb{R}\right\},
\end{gathered}
$$

where $(x, u) \in T M$, such that $T_{(x, u)} T M=\mathcal{H}_{(x, u)} \oplus \mathcal{V}_{(x, u)}$.

Let $X=X^{i} \frac{\partial}{\partial x^{i}}$ be a local vector field on $M$. The vertical and the horizontal lifts of $X$ are defined by

$$
\begin{aligned}
& X^{V}=X^{i} \frac{\partial}{\partial y^{i}}, \\
& X^{H}=X^{i} \frac{\delta}{\delta x^{i}}=X^{i}\left\{\frac{\partial}{\partial x^{i}}-y^{j} \Gamma_{i j}^{k} \frac{\partial}{\partial y^{k}}\right\} .
\end{aligned}
$$

For consequences, we have $\left(\frac{\partial}{\partial x^{i}}\right)^{H}=\frac{\delta}{\delta x^{i}}$ and $\left(\frac{\partial}{\partial x^{i}}\right)^{V}=\frac{\partial}{\partial y^{i}}$, then $\left(\frac{\delta}{\delta x^{i}}, \frac{\partial}{\partial y^{i}}\right)_{i=\overline{1, m}}$ is a local adapted frame on $T T M$.

If $w=w^{i} \frac{\partial}{\partial x^{i}}+\bar{w}^{j} \frac{\partial}{\partial x^{j}} \in T_{(x, u)} T M$, then its horizontal and vertical parts are defined by

$$
\begin{gathered}
w^{h}=w^{i} \frac{\partial}{\partial x^{i}}-w^{i} u^{j} \Gamma_{i j}^{k} \frac{\partial}{\partial y^{k}} \in \mathcal{H}_{(x, u)}, \\
w^{v}=\left(\bar{w}^{k}+w^{i} u^{j} \Gamma_{i j}^{k}\right) \frac{\partial}{\partial y^{k}} \in \mathcal{V}_{(x, u)} .
\end{gathered}
$$

Lemma 2.1 [15] Let $(M, g)$ be a Riemannian manifold and $R$ its tensor curvature, then for all vector fields $X, Y \in \Gamma(T M)$ we have:

$$
\begin{aligned}
& 1\left[X^{H}, Y^{H}\right]_{p}=[X, Y]_{p}^{H}-\left(R_{x}(X, Y) u\right)^{V}, \\
& 2\left[X^{H}, Y^{V}\right]_{p}=\left(\nabla_{X} Y\right)_{p}^{V}, \\
& 3\left[X^{V}, Y^{V}\right]_{p}=0,
\end{aligned}
$$

where $p=(x, u) \in T M$.

\section{Horizontal Sasaki gradient metric}

Definition 3.1 Let $(M, g)$ be a Riemannian manifold and $f: M \rightarrow] 0,+\infty[$ be a strictly positive smooth function on $M$. On the tangent bundle $T M$, we define a horizontal Sasaki gradient metric noted $g_{f}^{H}$ by

$$
\begin{array}{ll}
1 & g_{f}^{H}\left(X^{H}, Y^{H}\right)_{(x, u)}=g_{x}(X, Y)+X_{x}(f) Y_{x}(f), \\
2 & g_{f}^{H}\left(X^{H}, Y^{V}\right)_{(x, u)}=0, \\
3 & g_{f}^{H}\left(X^{V}, Y^{V}\right)_{(x, u)}=g_{x}(X, Y),
\end{array}
$$

where $X, Y \in \Gamma(T M),(x, u) \in T M$.

Remark 3.1 1 If $f$ be a constant then $g_{f}^{H}$ is the Sasaki metric [15], $2 g_{f}^{H}\left(X^{V},(\operatorname{grad} f)^{V}\right)=g(X, \operatorname{grad} f)=X(f)$, 
$3 g_{f}^{H}\left(X^{H},(\operatorname{grad} f)^{H}\right)=\left(1+\|\operatorname{grad} f\|^{2}\right) X(f)=\alpha X(f)$,

where $X, Y \in \Gamma(T M)$ and $\alpha=1+\|\operatorname{grad} f\|^{2},\|\cdot\|$ denote the norm with respect to $(M, g)$.

In the following, we consider $\alpha=1+\|\operatorname{grad} f\|^{2}$.

Lemma 3.1 Let $(M, g)$ be a Riemannian manifold, we have the following

$1 X^{V} g_{f}^{H}\left(Y^{V}, Z^{V}\right)=0$,

$2 X^{V} g_{f}^{H}\left(Y^{H}, Z^{H}\right)=0$,

$3 X^{H} g_{f}^{H}\left(Y^{V}, Z^{V}\right)=X g(Y, Z)$,

$4 X^{H} g_{f}^{H}\left(Y^{H}, Z^{H}\right)=X g(Y, Z)+X(Y(f)) \cdot Z(f)+Y(f) \cdot X(Z(f))$,

where $X, Y, Z \in \Gamma(T M)$.

Proof. Lemma 3.1 follows from Definition 3.1.

We shall calculate the Levi-Civita connection $\nabla^{f}$ of $T M$ with horizontal Sasaki gradient metric $g_{f}^{H}$. This connection is characterized by the Koszul formula:

$$
\begin{aligned}
2 g_{f}^{H}\left(\nabla_{\widetilde{X}}^{f} \widetilde{Y}, \widetilde{Z}\right)= & \widetilde{X} g_{f}^{H}(\widetilde{Y}, \widetilde{Z})+\widetilde{Y} g_{f}^{H}(\widetilde{Z}, \widetilde{X})-\widetilde{Z} g_{f}^{H}(\widetilde{X}, \widetilde{Y}) \\
& +g_{f}^{H}(\widetilde{Z},[\widetilde{X}, \widetilde{Y}])+g_{f}^{H}(\widetilde{Y},[\widetilde{Z}, \widetilde{X}])-g_{f}^{H}(\widetilde{X},[\widetilde{Y}, \widetilde{Z}]) .
\end{aligned}
$$

for all $\widetilde{X}, \widetilde{Y}, \widetilde{Z} \in \Gamma(T M)$.

Lemma 3.2 Let $(M, g)$ be a Riemannian manifold and $\left(T M, g_{f}^{H}\right)$ its tangent bundle equipped with the horizontal Sasaki gradient metric. If $\nabla\left(\right.$ resp $\left.\nabla^{f}\right)$ denote the Levi-Civita connection of $(M, g)\left(\operatorname{resp}\left(T M, g_{f}^{H}\right)\right)$, then we have:

1) $g_{f}^{H}\left(\nabla_{X^{H}}^{f} Y^{H}, Z^{H}\right)=g_{f}^{H}\left(\left(\nabla_{X} Y\right)^{H}+\frac{1}{\alpha} g\left(Y, \nabla_{X} \operatorname{grad} f\right)(\operatorname{grad} f)^{H}, Z^{H}\right)$,

2) $g_{f}^{H}\left(\nabla_{X^{H}}^{f} Y^{H}, Z^{V}\right)=-\frac{1}{2} g_{f}^{H}\left((R(X, Y) u)^{V}, Z^{V}\right)$,

3) $g_{f}^{H}\left(\nabla_{X^{H}}^{f} Y^{V}, Z^{H}\right)=-\frac{1}{\alpha} g(R(u, Y) X, \operatorname{grad} f) g_{f}^{H}\left((\operatorname{grad} f)^{H}, Z^{H}\right)$

$$
+\frac{1}{2} g_{f}^{H}\left((R(u, Y) X)^{H}, Z^{H}\right)
$$

4) $g_{f}^{H}\left(\nabla_{X^{H}}^{f} Y^{V}, Z^{V}\right)=g_{f}^{H}\left(\left(\nabla_{X} Y\right)^{V}, Z^{V}\right)$,

5) $g_{f}^{H}\left(\nabla_{X^{V}}^{f} Y^{H}, Z^{H}\right)=-\frac{1}{\alpha} g(R(u, X) Y, \operatorname{grad} f) g_{f}^{H}\left((\operatorname{grad} f)^{H}, Z^{H}\right)$

$$
+\frac{1}{2} g_{f}^{H}\left((R(u, X) Y)^{H}, Z^{H}\right)
$$

6) $g_{f}^{H}\left(\nabla_{X^{V}}^{f} Y^{H}, Z^{V}\right)=0$,

7) $g_{f}^{H}\left(\nabla_{X^{V}}^{f} Y^{V}, Z^{H}\right)=0$,

8) $g_{f}^{H}\left(\nabla_{X^{V}}^{f} Y^{V}, Z^{V}\right)=0$.

for all vector fields $X, Y \in \Gamma(T M)$, where $R$ denote the curvature tensor of $(M, g)$. 
Proof. The proof of Lemma 3.2 follows directly from Kozul formula (3.1), Lemma 2.1, Definition 3.1 and Lemma 3.1.

1) The statement is obtained as follows.

$$
\begin{aligned}
2 g_{f}^{H}\left(\nabla_{X^{H}}^{f} Y^{H}, Z^{H}\right)= & X^{H} g_{f}^{H}\left(Y^{H}, Z^{H}\right)+Y^{H} g_{f}^{H}\left(Z^{H}, X^{H}\right)-Z^{H} g_{f}^{H}\left(X^{H}, Y^{H}\right) \\
& +g_{f}^{H}\left(Z^{H},\left[X^{H}, Y^{H}\right]\right)+g_{f}^{H}\left(Y^{H},\left[Z^{H}, X^{H}\right]\right) \\
& -g_{f}^{H}\left(X^{H},\left[Y^{H}, Z^{H}\right]\right) \\
= & X g(Y, Z)+X(Y(f)) \cdot Z(f)+Y(f) \cdot X(Z(f))+Y g(Z, X) \\
& +Y(Z(f)) \cdot X(f)+Z(f) \cdot Y(X(f))-Z g(X, Y) \\
& -Z(X(f)) \cdot Y(f)-X(f) \cdot Z(Y(f))+g_{f}^{H}\left(Z^{H},[X, Y]^{H}\right) \\
& +g_{f}^{H}\left(Y^{H},[Z, X]^{H}\right)-g_{f}^{H}\left(X^{H},[Y, Z]^{H}\right) \\
= & X g(Y, Z)+X(Y(f)) \cdot Z(f)+Y g(Z, X)+Z(f) \cdot Y(X(f)) \\
& -Z g(X, Y)+g(Z,[X, Y])+Z(f) \cdot[X, Y](f) \\
& +g(Y,[Z, X])-g(X,[Y, Z]) \\
= & 2 g\left(\nabla_{X} Y, Z\right)+2 X(Y(f)) \cdot Z(f) \\
= & 2\left[g_{f}^{H}\left(\left(\nabla_{X} Y\right)^{H}, Z^{H}\right)-\nabla_{X} Y(f) \cdot Z(f)+X(Y(f)) \cdot Z(f)\right]
\end{aligned}
$$

which implies that

$$
\begin{aligned}
2 g_{f}^{H}\left(\nabla_{X^{H}}^{f} Y^{H}, Z^{H}\right) & =2\left[g_{f}^{H}\left(\left(\nabla_{X} Y\right)^{H}, Z^{H}\right)+\frac{1}{\alpha} g\left(Y, \nabla_{X} \operatorname{grad} f\right) g_{f}^{H}\left((\operatorname{grad} f)^{H}, Z^{H}\right)\right] \\
& =2 g_{f}^{H}\left(\left(\nabla_{X} Y\right)^{H}+\frac{1}{\alpha} g\left(Y, \nabla_{X} \operatorname{grad} f\right)(\operatorname{grad} f)^{H}, Z^{H}\right) .
\end{aligned}
$$

2) Direct calculations give

$$
\begin{aligned}
2 g_{f}^{H}\left(\nabla_{X^{H}}^{f} Y^{H}, Z^{V}\right)= & X^{H} g_{f}^{H}\left(Y^{H}, Z^{V}\right)+Y^{H} g_{f}^{H}\left(Z^{V}, X^{H}\right)-Z^{V} g_{f}^{H}\left(X^{H}, Y^{H}\right) \\
& +g_{f}^{H}\left(Z^{V},\left[X^{H}, Y^{H}\right]\right)+g_{f}^{H}\left(Y^{H},\left[Z^{V}, X^{H}\right]\right) \\
& -g_{f}^{H}\left(X^{H},\left[Y^{H}, Z^{V}\right]\right) \\
= & g_{f}^{H}\left(Z^{V},\left[X^{H}, Y^{H}\right]\right) \\
= & -g_{f}^{H}\left((R(X, Y) u)^{V}, Z^{V}\right)
\end{aligned}
$$

The other formulas are obtained by a similar calculation.

As a direct consequence of Lemma 3.2, we get the following theorem.

Theorem 3.1 Let $(M, g)$ be a Riemannian manifold and $\left(T M, g_{f}^{H}\right)$ its tangent bundle equipped with the horizontal Sasaki gradient metric. If $\nabla\left(\right.$ resp $\left.\nabla^{f}\right)$ denote the Levi-Civita 
connection of $(M, g)\left(\operatorname{resp}\left(T M, g_{f}^{H}\right)\right)$, then we have:

1. $\left(\nabla_{X^{H}}^{f} Y^{H}\right)_{p}=\left(\nabla_{X} Y\right)_{p}^{H}+\frac{1}{\alpha} g_{x}\left(Y, \nabla_{X} \operatorname{grad} f\right)(\operatorname{grad} f)_{P}^{H}$

$$
-\frac{1}{2}\left(R_{x}(X, Y) u\right)^{V}
$$

2. $\left(\nabla_{X^{H}}^{f} Y^{V}\right)_{p}=\left(\nabla_{X} Y\right)_{p}^{V}-\frac{1}{2 \alpha} g_{x}(R(u, Y) X, \operatorname{grad} f)(\operatorname{grad} f)_{p}^{H}$

$$
+\frac{1}{2}\left(R_{x}(u, Y) X\right)^{H},
$$

3. $\left(\nabla_{X^{V}}^{f} Y^{H}\right)_{p}=\frac{1}{2}\left(R_{x}(u, X) Y\right)^{H}-\frac{1}{2 \alpha} g_{x}(R(u, X) Y, \operatorname{grad} f)(\operatorname{grad} f)_{p}^{H}$,

4. $\left(\nabla_{X^{V}}^{f} Y^{V}\right)_{p}=0$,

for all vector fields $X, Y \in \Gamma(T M)$ and $p=(x, u) \in T M$, where $R$ denote the curvature tensor of $(M, g)$.

\section{Geodesics of horizontal Sasaki gradient metric.}

Let $(M, g)$ be a Riemannian manifold and $x: I \rightarrow M$ be a curve on $M$. We define a curve $C: I \rightarrow T M$ by for all $t \in I, C(t)=(x(t), y(t))$ where $y(t) \in T_{x(t)} M$ i.e $y(t)$ is a vector field along $x(t)$.

Definition 4.1 [12], [15] Let $(M, g)$ be a Riemannian manifold, if $x(t)$ is a curve on $M$. The curve $C(t)=(x(t), \dot{x}(t))$ is called the natural lift of curve $x(t)$.

Definition 4.2 [15] Let $(M, g)$ be a Riemannian manifold and $\nabla$ denote the Levi-Civita connection of $(M, g)$. A curve $C(t)=(x(t), \dot{x}(t))$ is said to be a horizontal lift of the curve $x(t)$ if and only if $\nabla_{\dot{x}} y=0$.

Lemma 4.1 [16] Let $(M, g)$ be a Riemannian manifold. If $X, Y \in \Gamma(T M)$ are vector fields on $M$ and $(x, u) \in T M$ such that $Y_{x}=u$, then we have:

$$
d_{x} Y\left(X_{x}\right)=X_{(x, u)}^{H}+\left(\nabla_{X} Y\right)_{(x, u)}^{V}
$$

Proof. Let $\left(U, x^{i}\right)$ be a local chart on $M$ in $x \in M$ and $\left.\pi^{-1}(U), x^{i}, y^{j}\right)$ be the induced chart on $T M$, if $X_{x}=\left.X^{i}(x) \frac{\partial}{\partial x^{i}}\right|_{x}$ and $Y_{x}=\left.Y^{i}(x) \frac{\partial}{\partial x^{i}}\right|_{x}=u$, then

$$
d_{x} Y\left(X_{x}\right)=\left.X^{i}(x) \frac{\partial}{\partial x^{i}}\right|_{(x, u)}+\left.X^{i}(x) \frac{\partial Y^{k}}{\partial x^{i}}(x) \frac{\partial}{\partial y^{k}}\right|_{(x, u)}
$$

thus the horizontal part is given by:

$$
\begin{aligned}
\left(d_{x} Y\left(X_{x}\right)\right)^{h} & =\left.X^{i}(x) \frac{\partial}{\partial x^{i}}\right|_{(x, u)}-\left.X^{i}(x) Y^{j}(x) \Gamma_{i j}^{k}(x) \frac{\partial}{\partial y^{k}}\right|_{(x, u)} \\
& =X_{(x, u)}^{h}
\end{aligned}
$$

and the vertical part is given by:

$$
\begin{aligned}
\left(d_{x} Y\left(X_{x}\right)\right)^{v} & =\left.\left\{X^{j}(x) \frac{\partial Y^{k}}{\partial x^{i}}(x)+X^{i}(x) Y^{j}(x) \Gamma_{i j}^{k}(x)\right\} \frac{\partial}{\partial y^{k}}\right|_{(x, u)} \\
& =\left(\nabla_{X} Y\right)_{(x, u)}^{v}
\end{aligned}
$$


Lemma 4.2 [16] Let $(M, g)$ be a Riemannian manifold and $\nabla$ denote the Levi-Civita connection of. If $x(t)$ be a curve on $M$ and $C(t)=(x(t), y(t))$ be a curve on $T M$, then

$$
\dot{C}=\dot{x}^{H}+\left(\nabla_{\dot{x}} y\right)^{V} .
$$

Proof. Locally, if $Y \in \Gamma(T M)$ is a vector field such $Y(x(t))=y(t)$, then we have

$$
\dot{C}(t)=d C(t)=d Y(x(t)) .
$$

Using Lemma 4.1 we obtain

$$
\dot{C}(t)=d Y(x(t))=\dot{x}^{H}+\left(\nabla_{\dot{x}} y\right)^{V} .
$$

Theorem 4.1 Let $(M, g)$ be a Riemannian manifold and $\left(T M, g_{f}^{H}\right)$ its tangent bundle equipped with the horizontal Sasaki gradient metric. If $\nabla\left(\right.$ resp. $\left.\nabla^{f}\right)$ denote the Levi-Civita connection of $(M, g)$ (resp. $\left.\left(T M, g_{f}^{H}\right)\right)$ and $C(t)=(x(t), y(t))$ is the curve on TM such $y(t)$ is a vector field along $x(t)$, then

$$
\begin{aligned}
\nabla_{\dot{C}}^{f} \dot{C}= & \left(\nabla_{\dot{x}} \dot{x}\right)^{H}+\frac{1}{\alpha}\left[g\left(\dot{x}, \nabla_{\dot{x}} g r a d f\right)-g\left(R\left(y, \nabla_{\dot{x}} y\right) \dot{x}, \operatorname{grad} f\right)\right](\operatorname{grad} f)^{H} \\
& +\left(R\left(y, \nabla_{\dot{x}} y\right) \dot{x}\right)^{H}+\left(\nabla_{\dot{x}} \nabla_{\dot{x}} y\right)^{V}
\end{aligned}
$$

Proof. Using Lemma 4.2 we obtain

$$
\begin{aligned}
\nabla_{\dot{C}}^{f} \dot{C}= & \left.\nabla_{\left[\dot{x}^{H}\right.}^{f}+\left(\nabla_{\dot{x}} y\right)^{V}\right] \\
= & \left.\nabla_{\dot{x}^{H}}^{f} \dot{x}^{H}+\nabla_{\dot{x}^{H}}^{f}\left(\nabla_{\dot{x}} y\right)^{V}+\nabla_{\dot{x}}^{f}\right)_{\left.\left(\nabla_{\dot{x}} y\right)^{V}\right]} \dot{x}^{H}+\nabla_{\left(\nabla_{\dot{x}} y\right)^{V}}^{f}\left(\nabla_{\dot{x}} y\right)^{V} \\
= & \left(\nabla_{\dot{x}} \dot{x}\right)^{H}+\frac{1}{\alpha} g\left(\dot{x}, \nabla_{\dot{x}} g r a d f\right)(\operatorname{grad} f)^{H}-\frac{1}{2}(R(\dot{x}, \dot{x}) y)^{V} \\
& +\left(\nabla_{\dot{x}} \nabla_{\dot{x}} y\right)^{V}-\frac{1}{2 \alpha} g\left(R\left(y, \nabla_{\dot{x}} y\right) \dot{x}, \operatorname{grad} f\right)(\operatorname{grad} f)^{H} \\
& +\frac{1}{2}\left(R\left(y, \nabla_{\dot{x}} y\right) \dot{x}\right)^{H}-\frac{1}{2 \alpha} g\left(R\left(y, \nabla_{\dot{x}} y\right) \dot{x}, \operatorname{grad} f\right)(\operatorname{grad} f)^{H} \\
& +\frac{1}{2}\left(R\left(y, \nabla_{\dot{x}} y\right) \dot{x}\right)^{H} \\
= & \left(\nabla_{\dot{x}} \dot{x}\right)^{H}+\frac{1}{\alpha}\left[g\left(\dot{x}, \nabla_{\dot{x}} g^{\operatorname{rad} f}\right)-g\left(R\left(y, \nabla_{\dot{x}} y\right) \dot{x}, \operatorname{grad} f\right)\right](\operatorname{grad} f)^{H} \\
& +\left(R\left(y, \nabla_{\dot{x}} y\right) \dot{x}\right)^{H}+\left(\nabla_{\dot{x}} \nabla_{\dot{x}} y\right)^{V} .
\end{aligned}
$$

Theorem 4.2 Let $(M, g)$ be a Riemannian manifold and $\left(T M, g_{f}^{H}\right)$ its tangent bundle equipped with the horizontal Sasaki gradient metric. If $C(t)=(x(t), y(t))$ is curve on $T M$ such $y(t)$ is a vector field along $x(t)$, then $C(t)$ is a geodesic on TM if and only if

$$
\left\{\begin{array}{l}
\nabla_{\dot{x}} \dot{x}=\frac{1}{\alpha}\left[g\left(R\left(y, \nabla_{\dot{x}} y\right) \dot{x}, \operatorname{grad} f\right)-g\left(\dot{x}, \nabla_{\dot{x}} g r a d f\right)\right] \operatorname{grad} f-R\left(y, \nabla_{\dot{x}} y\right) \dot{x}, \\
\nabla_{\dot{x}} \nabla_{\dot{x}} y=0 .
\end{array}\right.
$$

Proof. The statement is a direct consequence of Theorem 4.1 and definition of geodesic.

Using Theorem 4.2 we deduce: 
Corollary 4.1 Let $(M, g)$ be a Riemannian manifold and $\left(T M, g_{f}^{H}\right)$ its tangent bundle equipped with the horizontal Sasaki gradient metric. The natural lift $C(t)=(x(t), \dot{x}(t))$ of any geodesic $x(t)$ is a geodesic on $T M$ if and only if

$$
\nabla_{\dot{x}} \operatorname{grad} f=0 \text { or } \operatorname{grad} f=0 \text {. }
$$

Corollary 4.2 Let $(M, g)$ be a Riemannian manifold, $\left(T M, g_{f}^{H}\right)$ its tangent bundle equipped with the horizontal Sasaki gradient metric. The horizontal lift $C(t)=(x(t), y(t))$ of the curve $x(t)$ is a geodesic on $T M$ if and only if

$$
\nabla_{\dot{x}} \dot{x}=-\frac{1}{\alpha} g\left(\dot{x}, \nabla_{\dot{x}} \operatorname{grad} f\right) \operatorname{grad} f .
$$

Corollary 4.3 Let $(M, g)$ be a Riemannian manifold, $\left(T M, g_{f}^{H}\right)$ its tangent bundle equipped with the horizontal Sasaki gradient metric. The horizontal lift $C(t)=(x(t), y(t))$ of any geodesic $x(t)$ is a geodesic on $T M$ if and only if

$$
\nabla_{\dot{x}} \operatorname{grad} f=0 \text { or } \operatorname{grad} f=0 .
$$

Remark 4.1 Let $\left(M^{m}, g\right)$ be an m-dimensional Riemannian manifold. If $C(t)=(x(t), y(t))$ is a horizontal lift of the curve $x(t)$, locally we have:

$$
\nabla_{\dot{x}} y=0 \Leftrightarrow \frac{d y^{k}}{d t}+\Gamma_{i j}^{k} y^{i} \frac{d x^{j}}{d t}=0 \Leftrightarrow y^{\prime}(t)=A(t) \cdot y(t),
$$

where, $A(t)=\left[a_{k j}\right], a_{k j}=\sum_{i=1}^{m}-\Gamma_{i j}^{k} \frac{d x^{j}}{d t}$.

Remark 4.2 Using the Remark 4.1 we can costruct an infinity of examples of geodesics on $\left(T M, g^{f}\right)$.

Example 1 We consider on $\mathbb{R}$ the metric $g=e^{x} d x^{2}$.

The Christoffel symbols of the Levi-cita connection associated with $g$ are

$$
\Gamma_{11}^{1}=\frac{1}{2} g^{11}\left(\frac{\partial g_{11}}{\partial x^{1}}+\frac{\partial g_{11}}{\partial x^{1}}-\frac{\partial g_{11}}{\partial x^{1}}\right)=\frac{1}{2} .
$$

1) The geodesics $x(t)$ such that $x(0)=a \in \mathbb{R}, x^{\prime}(0)=v \in \mathbb{R}$ of $g$ satisfy the equation,

$$
\frac{d^{2} x^{k}}{d t^{2}}+\sum_{i, j=1}^{n} \frac{d x^{i}}{d t} \frac{d x^{j}}{d t} \Gamma_{i j}^{j}=0 \Leftrightarrow x^{\prime \prime}+\frac{1}{2}\left(x^{\prime}\right)^{2}=0 .
$$

Hence $x^{\prime}(t)=\frac{2 v}{2+v t}, x(t)=a+2 \ln \left(1+\frac{v t}{2}\right)$ and $\dot{x}(t)=x^{\prime}(t) \frac{d}{d x}$.

Then $C_{1}(t)=(x(t), \dot{x}(t))=\left(a+2 \ln \left(1+\frac{v t}{2}\right), \frac{2 v}{2+v t} \frac{d}{d x}\right)$ is a natural lift on $T \mathbb{R}$.

2 ) The curve $C_{2}(t)=(x(t), y(t))$ such $\nabla_{\dot{x}} y=0$ satisfy the equation,

$$
\frac{d y^{s}}{d t}+y^{i} \Gamma_{i j}^{s} \frac{d x^{j}}{d t}=0 \Leftrightarrow y^{\prime}+\frac{1}{2} y x^{\prime}=0,
$$

after that $y(t)=k \cdot \exp \left(\frac{-v}{2+t v}\right) \frac{d}{d x}, k \in \mathbb{R}$.

Then $C_{2}(t)=(x(t), y(t))=\left(a+2 \ln \left(1+\frac{v t}{2}\right), k \cdot \exp \left(\frac{-v}{2+t v}\right) \frac{d}{d x}\right)$ is a horizontal lift on 
$T \mathbb{R}$.

3) We study the condition

$$
\nabla_{\dot{x}} \operatorname{grad} f=0 \text { or } \operatorname{grad} f=0 .
$$

i) If $\operatorname{grad} f=0$ i.e $f$ is constant, from Corollary 4.1 and Corollary 4.3 , the curves $C_{1}(t)$ and $C_{2}(t)$ are geodesics on $T \mathbb{R}$.

ii) If $f$ is non constant and

$$
\nabla_{\dot{x}} \operatorname{grad} f=0 \Leftrightarrow \nabla_{x^{\prime}(t) \frac{d}{d x}} e^{-x} f^{\prime}(x) \frac{d}{d x}=0 \Leftrightarrow f^{\prime \prime}(x)-\frac{1}{2} f^{\prime}(x)=0,
$$

after that $f(x)=2 k_{1}$. $\exp \left(\frac{x}{2}\right)+k_{2}, k_{1}, k_{2} \in \mathbb{R}, k_{1} \neq 0$.

From Corollary 4.1 and Corollary 4.3 , the curves $C_{1}(t)$ and $C_{2}(t)$ are geodesics on $T \mathbb{R}$.

Example 2 Consider the upper half-plane

$$
\mathbb{R}_{+}^{2}=\left\{(x, y) \in \mathbb{R}^{2}, y>0\right\},
$$

equipped withe the Riemannian metric $g$ define by

$$
g_{11}=1, g_{22}=y^{2}, g_{12}=g_{21}=0 .
$$

The Christoffel symbols of the Levi-cita connection are given by:

$$
\Gamma_{11}^{1}=\Gamma_{12}^{1}=\Gamma_{21}^{1}=\Gamma_{22}^{1}=0, \Gamma_{11}^{2}=\Gamma_{12}^{2}=\Gamma_{21}^{2}=0, \Gamma_{22}^{2}=\frac{1}{y} .
$$

Let $C(t)=(x(t), y(t), u(t), v(t))$ is horizontal lift of the curve $(x(t), y(t))$, the matrix $A(t)$ is given by

$$
A(t)=\left(\begin{array}{cc}
0 & 0 \\
0 & -\frac{y^{\prime}(t)}{y(t)}
\end{array}\right),
$$

and

$$
(u(t), v(t))=\left(k_{1}, \frac{k_{2}}{y(t)}\right),
$$

then $C(t)=\left(x(t), y(t), k_{1}, \frac{k_{2}}{y(t)}\right), k_{1}, k_{2} \in \mathbb{R}$.

Corollary 4.4 Let $(M, g)$ be a Riemannian manifold and $\left(T M, g_{f}^{H}\right)$ its tangent bundle equipped with the horizontal Sasaki gradient metric. If grad $f=0$, then $C(t)=(x(t), y(t))$ is a geodesic on $T M$ if and only if

$$
\left\{\begin{array}{l}
\nabla_{\dot{x}} \dot{x}=-R\left(y, \nabla_{\dot{x}} y\right) \dot{x} \\
\nabla_{\dot{x}} \nabla_{\dot{x}} y=0
\end{array}\right.
$$

Proof. The statement is a direct consequence of Theorem 4.1.

Theorem 4.3 Let $(M, g)$ be a Riemannian manifold, (TM, $\left.g_{f}^{H}\right)$ its tangent bundle equipped with the horizontal Sasaki gradient metric and $x(t)$ be a geodesic on $M$. If $C(t)=(x(t), y(t))$ is a geodesic on $T M$ such that grad $f \neq 0$ then:

$$
g\left(R\left(y, \nabla_{\dot{x}} y\right) \dot{x}, \operatorname{grad} f\right)=g\left(\dot{x}, \nabla_{\dot{x}} \operatorname{grad} f\right)
$$


Proof. Let $x(t)$ be a geodesic on $M$, then $\nabla_{\dot{x}} \dot{x}=0$. Using the first equation of formula (4.3) we obtain

$$
\begin{aligned}
g\left(\nabla_{\dot{x}} \dot{x}, \dot{x}\right)=0 \Rightarrow & \frac{1}{\alpha}\left[g\left(R\left(y, \nabla_{\dot{x}} y\right) \dot{x}, \operatorname{grad} f\right)-g\left(\dot{x}, \nabla_{\dot{x}} g r a d f\right)\right] g(\operatorname{grad} f, \dot{x}) \\
& -g\left(R\left(y, \nabla_{\dot{x}} y\right) \dot{x}, \dot{x}\right)=0 \\
\Rightarrow & g\left(R\left(y, \nabla_{\dot{x}} y\right) \dot{x}, \operatorname{grad} f\right)=g\left(\dot{x}, \nabla_{\dot{x}} g r a d f\right) .
\end{aligned}
$$

Theorem 4.4 Let $(M, g)$ be a flat Riemannian manifold and $\left(T M, g_{f}^{H}\right)$ its tangent bundle equipped with the horizontal Sasaki gradient metric. Then the curve $C(t)=(x(t), y(t))$ is a geodesic on $T M$ if and only if

$$
\left\{\begin{array}{l}
\nabla_{\dot{x}} \dot{x}=-\frac{1}{\alpha} g\left(\dot{x}, \nabla_{\dot{x}} \operatorname{grad} f\right) \operatorname{grad} f, \\
\nabla_{\dot{x}} \nabla_{\dot{x}} y=0 .
\end{array}\right.
$$

Proof. The statement is a direct consequence of Theorem 4.1.

Corollary 4.5 Let $(M, g)$ be a flat Riemannian manifold and $\left(T M, g_{f}^{H}\right)$ its tangent bundle equipped with the horizontal Sasaki gradient metric. If $\nabla_{\dot{x}}$ grad $f=0$, then the curve $C(t)=(x(t), y(t))$ is a geodesic on $T M$ if and only if $x(t)$ is a geodesic on $M$ and $\nabla_{\dot{x}} \nabla_{\dot{x}} y=0$.

Proof. The statement is a direct consequence of Theorem 4.4.

\section{References}

1. Abbassi, M.T.K., Sarih, M.: On Natural Metrics on Tangent Bundles of Riemannian Manifolds, Arch. Math. (Brno) 41, 71-92 (2005).

2. Belarbi, L., El Hendi, H., Latti, F.: On the geometry of the tangent bundle with vertical rescaled generalized Cheeger-Gromoll metric,Bull. Transilv. Univ. Brasov Ser. III 12 (61), 247-264 (2019).

3. Cengiz, N., Salimov, A.A.: Diagonal lift in the tensor bundle and its applications, Appl. Math. Comput. 142 (2-3), 309-319 (2003).

4. Djaa, M. and Gancarzewicz, J.: The geometry of tangent bundles of order $r$, Boletin Academia, Galega de Ciencias, 4, 147-165 (1985).

5. Dombrowski, P.: On the Geometry of the Tangent Bundle, J. Reine Angew. Math. 210, 73-88 (1962).

6. Gudmundsson, S., Kappos, E.: On the Geometry of the Tangent Bundle with the Cheeger-Gromoll Metric, Tokyo J. Math. 25 (1), 75-83 (2002).

7. Kada Ben Otmane, R., Zagane, A., Djaa, M.: On generalized cheeger-gromoll metric and harmonicity, Commun. Fac. Sci. Univ. Ank. Ser. A1 Math. Stat. 69 (1), 629-645 (2020).

8. Musso, E., Tricerri, F.: Riemannian Metrics on Tangent Bundles, Ann. Mat. Pura. Appl. 150 (4), 1-19 (1988).

9. Salimov, A.A., Gezer, A., Akbulut, K.: Geodesics of Sasakian metrics on tensor bundles, Mediterr. J. Math. 6 (2), 135-147 (2009).

10. Salimov, A.A, Gezer, A.: On the geometry of the $(1,1)$-tensor bundle with Sasaki type metric, Chin. Ann. Math. Ser. B, 32 (3), 369-386 (2011).

11. Salimov, A.A., Agca, F.: Some Properties of Sasakian Metrics in Cotangent Bundles, Mediterr. J. Math. 8 (2), 243-255 (2011). 
12. Salimov, A.A., Kazimova, S.: Geodesics of the Cheeger-Gromoll Metric, Turkish J. Math. 33, 99-105 (2009).

13. Sasaki, S.: On the differential geometry of tangent bundles of Riemannian manifolds II, Tohoku Math. J. 10, 338-354 (1958).

14. Sekizawa, M.: Curvatures of Tangent Bundles with Cheeger-Gromoll Metric, Tokyo J. Math. 14 (2), 407-417 (1991).

15. Yano, K., Ishihara, S.: Tangent and Cotangent Bundles. Marcel Dekker. INC. New York (1973).

16. Zagane, A., Djaa, M.: On Geodesics of Warped Sasaki Metric, Math. Sci. Appl. ENotes 5 (1), 85-92 (2017).

17. Zagane, A., Djaa, M.: Geometry of Mus-Sasaki metric, Commun. Math. 26 (2), $113-$ 126 (2018). 